\title{
Obscure Gastrointestinal Bleeding: A Diagnostic Dilemma?
}

CrossMark

\section{Ali Ghavidel 1,**}

1. Assistant Professor, Liver and Gastrointestinal Diseases Research Centre, Imam Reza Hospital, Tabriz University of Medical Sciences, Tabriz, Iran

* Corresponding Author:

Ali Ghavidel, MD

Tabriz University of Medical Sciences, Liver and Gastrointestinal Diseases Research Centre, Imam Reza Hospital, Tabriz, Iran Telefax: + 984133347554

Email: ali.ghavidel3@gmail.com

Received: 01 Feb. 2020

Accepted: 06 Jun. 2020

\section{Please cite this paper as:}

Ghavidel A. An Uncommon Cause of Upper Gastrointestinal Bleeding? Or Obscure Gastrointestinal Bleeding: A Diagnostic Dilemma? Middle East J Dig Dis 2020;12:214-216. doi: 10.34172/ mejdd.2020.185.
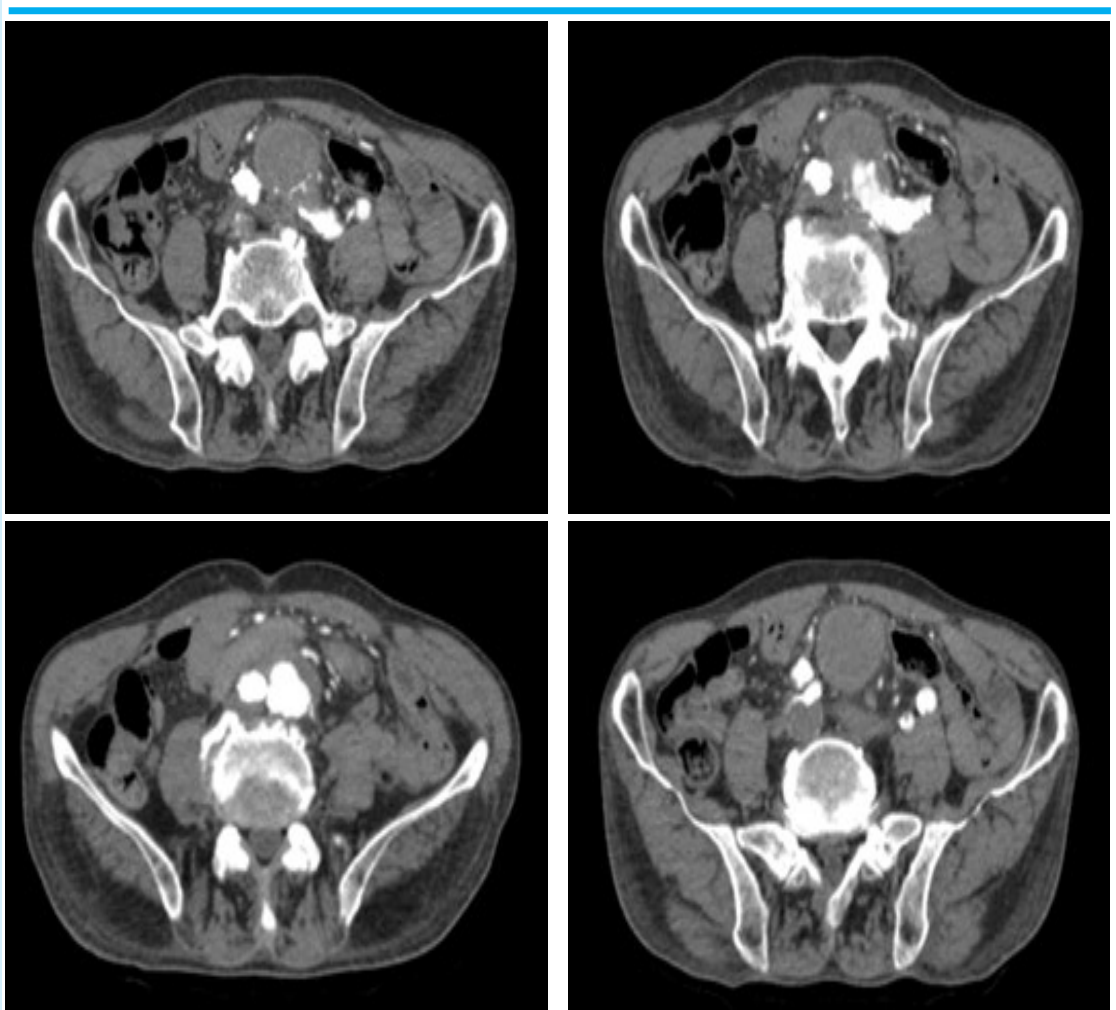

Fig.1: Abdominal and pelvic computed tomogram with and without contrast agent showed a 5-cm saccular aneurysmal mass around the bifurcation of the abdominal aorta that was associated with mural thrombosis and was extended to proximal common iliac artery. Surgical consultation was requested, and the surgeon transferred him to the operation room with an appropriate condition for surgery.

We present a case of obscure gastrointestinal bleeding with the clinical presentation of hematemesis and melena. The patient was a 68 -year-old man who complained of recurrent upper gastrointestinal bleeding and presented with hematemesis and melena. He provided no history of abdominal vascular reconstructive surgery, peptic ulcer disease, or any other diseases. On physical examination, the patient looked anemic with pale skin and was in an altered hemodynamic state. His vital signs were unstable. Heart and lungs examinations were normal. There was no scar on the abdomen. Epigastrium was tender on

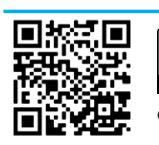


palpation without any organomegaly. He was admitted with the clinical presentation of upper gastrointestinal bleeding with hematemesis and melena. Laboratory evaluation, including blood tests, showed anemia. Emergency upper and lower endoscopy did not find a bleeding source. Total colonoscopy with terminal ileal intubation was negative. Abdominal and pelvic computed tomography (CT) with and without contrast showed a 5-cm saccular aneurysmal mass around the bifurcation of the abdominal aorta that was associated with mural thrombosis and was extended to proximal common iliac artery. The second part of the duodenum was attached to the upper border of aneurysmal mass with suspicious communication between the aorta and the small intestine.

What is your diagnosis?

\section{Answer:}

\section{Aorto-duodenal fistula}

The aneurysmal mass was resected, and vascular graft reconstruction was done. There was a defect in the upper wall of the aortic aneurysm contiguous with the fourth part of the duodenum. ${ }^{1,2}$ Abdominal and pelvic CT with and without contrast showed Increased enhancement material in the fourth part of the duodenum. Air bubbles of gas in the aneurysmal sac and periaortic inflammatory tissue were not seen. An aorto-duodenal fistula was found between the aorta and the fourth part of the duodenum that was ligated. ${ }^{3}$ And aneurysmal mass was resected with vascular graft re-construction.

This case illustrates the intensive care of life-threatening hemorrhage from an aorto-enteric fistula. This patient survived probably because of the rapid and correct diagnosis and also the team working between the gastroenterologist, the radiologist, and the surgeon.

\section{DISCUSSION}

The diagnosis and treatment of aorto-enteric fistula are difficult. It is a big diagnostic problem for gastroenterologists and surgeons. ${ }^{1}$ But in a patient with hematemesis and melena with aortic aneurysm when upper and lower endoscopy could not yield a bleeding source, a diagnosis of aorto-enteric fistula should be considered. ${ }^{2}$ Diagnosis of aorto-enteric fistula requires the physician to be highly suspicious about the patient presenting with signs and symptoms of gastrointestinal bleeding. In the management of acute gastrointestinal bleeding and aortoduodenal fistula endoscopy plays an major role. In hemodynamically stable patient. Endoscopy should only be performed when with suspection of aorto-enteric fistula. A normal endoscopy does not rule out diagnosis of aorto-enteric fistula, and realy it is not is not sensitive in the diagnosis of aorto-enteric fistula. Diagnosis of aorto-enteric fistula results from a highly clinical suspicion. The role of endoscopy is to rule out other etiologies of acute upper gastrointestinal bleeding, like peptic ulcers, esophageal varices. Enteroscope may be useful, but It should be remembered that obserevation of a fistula in the distal portion of upper part of small intestine is extremely difficult because of anatomic limitations. Consideration of the "herald bleed" provide time for correct diagnosis and treatment. Diagnosis in early stages is essential for a promising prognosis because of the fatal nature of aortoenteric fistula. Upper and lower endoscopy and spiral CT with dynamic angiography and with double contrast are the most useful methods to diagnose aorto-enteric fistula. ${ }^{3}$ Treatment requires surgical resection of the aneurysm and anatomic and physiological reconstruction. In primary aorto-enteric fistula anatomic reconstruction with a Dacron and with repair of the adjacent area of small bowel with insertion of omentum between the intestinal and aortic fistula, is practical method. Recent trials with one stage reconstruction have shown that some materials could be useful for one-stage revascularization with better prognosis. ${ }^{4}$ In our case, the available clinical, instrumental, and radiological supports made the hypothesis of such a diagnosis very probable. These findings, associated with gastroesophageal bleeding and the history of aortic aneurysm, led to the diagnosis of aorto-enteric fistula. The aim of this report was to emphasize the early diagnosis and management of all gastrointestinal bleedings in patients without a history of aortic aneurysm. ${ }^{5,6}$

\section{ACKNOWLEDGMENTS}

I am sincerely grateful to those who shared their truthful views on the article.

\section{ETHICAL APPROVAL}

There is nothing to be declared. 
CONFLICT OF INTEREST

The author declares no conflict of interest related to this work.

\section{REFERENCES}

1. Diethrich EB, Campbell DA, Brandt RL.Gastrointestinal hemorrhage. Presenting symptom of aortoduodenal fistulization. Am J Surg 1966;112:903-7. doi: 10.1016/00029610(66)90148-6

2. Reckless JP, McColl I, Taylor GW. Aorto-enteric fistulae: an uncommon complication of abdominal aortic aneurysms. Br J Surg 1972;59:458-60. doi: 10.1002/bjs.1800590613

3. Dachs RJ, Berman J. Aortoenteric fistula. Am Fam Physician 1992; 45:2610-6.

4. Chang MW, Chan Y, Hsieh H, Chang S. Secondary Aortoduodenal Fistula. Chang Gung Med J 2002;25:626-30.

5. Kayashima A, Mori H, Okuzawa A, Kubosawa Y, Hirai Y, Kinoshita S, et al. An Esophageal Ulcer Associated with a Thoracoabdominal Aortic Aneurysm. Case Rep Gastroenterol 2019;13:214-8. doi: 10.1159/000500067

6. Connolly JE, Kwaan JH, McCart PM, Brownell DA, Levine EF. Aortoenteric fistula. Ann Surg 1981;194:402412 doi: 10.1097/00000658-198110000-00004 